\title{
Thermal evolution of the Schwinger model with matrix product operators
}

\author{
M. C. Bañuls, ${ }^{1}$ K. Cichy, ${ }^{2-4}$ J. I. Cirac, ${ }^{1}$ K. Jansen, ${ }^{3}$ and H. Saito ${ }^{3}$ \\ ${ }^{1}$ Max-Planck-Institut für Quantenoptik, Hans-Kopfermann-Straße 1, 85748 Garching, Germany \\ ${ }^{2}$ Goethe-Universität, Institut für Theoretische Physik, Max-von-Laue-Straße 1, \\ D-60438 Frankfurt am Main, Germany \\ ${ }^{3}$ NIC, DESY, Platanenallee 6, D-15738 Zeuthen, Germany \\ ${ }^{4}$ Faculty of Physics, Adam Mickiewicz University, Umultowska 85, 61-614 Poznan, Poland
}

(Received 10 May 2015; published 28 August 2015)

\begin{abstract}
We demonstrate the suitability of tensor network techniques for describing the thermal evolution of lattice gauge theories. As a benchmark case, we have studied the temperature dependence of the chiral condensate in the Schwinger model, using matrix product operators to approximate the thermal equilibrium states for finite system sizes with nonzero lattice spacings. We show how these techniques allow for reliable extrapolations in bond dimension, step width, system size and lattice spacing, and for a systematic estimation and control of all error sources involved in the calculation. The reached values of the lattice spacing are small enough to capture the most challenging region of high temperatures and the final results are consistent with the analytical prediction by Sachs and Wipf over a broad temperature range.
\end{abstract}

DOI: $10.1103 /$ PhysRevD.92.034519

PACS numbers: 11.15.Ha, 02.70.-c

\section{INTRODUCTION}

Tensor network (TN) techniques have recently revealed their potential to study lattice gauge theories (LGT). Numerical studies have demonstrated that matrix product states (MPS) can accurately describe ground state and low excited levels of the Schwinger model [1-6] and of related quantum link models $[7,8]$, and tensor renormalization group methods have been used to evaluate the path integral $[9,10]$. More generally, a framework has been proposed to construct gauge invariant TN states in higher dimensions [11,12].

Thermal equilibrium states at arbitrary temperatures can be studied using a TN operator Ansatz, independent of the pure state approximations for low energy eigenstates. Nonzero temperature studies have played a major role in lattice QCD computations, establishing a crossover behavior of QCD in the early Universe [13]. Such calculations will be very important in the future for understanding QCD matter; see [14]. Different from most LGT calculations, TN methods work in the Hamiltonian formalism. In this approach, it is in principle possible to follow the complete thermal evolution of the system, and to map out the temperature dependence of physical quantities over a broad temperature regime.

In this work, we show that the matrix product operator (MPO) Ansatz can accurately describe the thermal equilibrium states of the Schwinger model. To this end, we investigate the temperature dependence of the chiral condensate in the continuum limit for the massless case, for which analytical results were provided in [15]. Our results are consistent with the analytical prediction at all (dimensionless) inverse temperatures $g \beta \in[0,6]$. Our lattice calculation using MPO requires a series of consecutive extrapolations. We describe how to carry out these steps and demonstrate that all systematic errors inherent to the method can be controlled and systematically improved. Thus, the procedure yields reliable continuum values and is applicable also when the exact value is completely unknown.

We use Gauss' law to integrate out the gauge degrees of freedom and apply TN states to describe the fermionic degrees of freedom in the exact physical subspace, as in $[3,4]$. Here we demonstrate that this approach, initially presented in [16], is also suitable for thermal states. Alternatively, one could include both fermionic and bosonic degrees of freedom and impose gauge symmetry on the tensors, as in $[1,5-8]$. In that case the gauge degrees of freedom need to be truncated, which introduces an additional extrapolation in the procedure, which also has to be taken into account in the systematic errors.

\section{THE MODEL AND THE HAMILTONIAN SETUP}

The Schwinger model [17] or QED in $1+1$ dimensions is frequently used as a test bench for lattice calculations. In order to apply TN methods, we work in the Hamiltonian formalism (see e.g. [3]), which implies we have to impose Gauss' law as a constraint on physical states. The Kogut-Susskind Hamiltonian [18] can be mapped to a spin system by a Jordan-Wigner transformation [19]. Using Gauss' law, the gauge degrees of freedom can be eliminated [20], since the electric flux on a link is completely determined by the spin content and the background field, so that the model can be written 


$$
\begin{aligned}
H= & x \sum_{n=0}^{N-2}\left[\sigma_{n}^{+} \sigma_{n+1}^{-}+\sigma_{n}^{-} \sigma_{n+1}^{+}\right]+\frac{\mu}{2} \sum_{n=0}^{N-1}\left[1+(-1)^{n} \sigma_{n}^{z}\right] \\
& +\sum_{n=0}^{N-2}\left[\ell+\frac{1}{2} \sum_{k=0}^{n}\left((-1)^{k}+\sigma_{k}^{z}\right)\right]^{2}
\end{aligned}
$$

where $\ell$ is the boundary electric field (on the leftmost link), which can describe the background field, and the parameters of the model are $x=\frac{1}{g^{2} a^{2}}, \mu=\frac{2 m}{g^{2} a}$, in terms of the lattice spacing, $a$, the fermion mass, $m$, and the coupling, $g$.

It is then possible to use a basis $|\ell\rangle\left|i_{0} i_{1} \ldots i_{N-1}\right\rangle$ to describe the physical space. For finite systems, the value of $\ell$ is conserved. In the following, we consider the case $\ell=0$ and omit it from the basis. We focus on the temperature dependence of the chiral condensate, $\Sigma=\langle\bar{\Psi} \Psi\rangle / g$, which is the order parameter of the chiral symmetry breaking, and which in terms of spin operators reads $\Sigma=\frac{\sqrt{x}}{N} \sum_{n}(-1)^{n} \frac{1+\sigma_{n}^{z}}{2}$.

\section{THE ANSATZ}

An MPS for a system of $N$ sites with internal dimension $d$ and individual basis $\{|i\rangle\}_{i=1}^{d}$ is a state of the form $|\Psi\rangle=\sum_{i_{0}, \ldots i_{N-1}=1}^{d} \operatorname{tr}\left(A_{0}^{i_{0}} \ldots A_{N-1}^{i_{N-1}}\right)\left|i_{0}, \ldots i_{N-1}\right\rangle$, where each $A_{k}^{i}$ is a $D$-dimensional matrix, and the bond dimension, $D$, determines the number of free parameters in the Ansatz [21-23]. MPS are known to provide good approximations to ground states of local Hamiltonians in the gapped phase [24] and have also been successfully used for more general situations [25]. The analogous Ansatz in the space of operators [26-28] is called MPO, and can be used to efficiently approximate thermal states of local Hamiltonians $[29,30]$.

To find an MPO approximation to the Gibbs state, $\rho \propto e^{-\beta H}$, a Suzuki-Trotter decomposition is applied to the exponential, with the Hamiltonian split into several terms whose exponentials are easily written or approximated as MPO. In the case of Hamiltonian (1), it is convenient to split $H=H_{e}+H_{o}+H_{z}$, where $H_{e(o)}$ contains the $\sigma_{n}^{+} \sigma_{n+1}^{-}+$H.c. terms for even (odd) $n$, and $H_{z}$ contains the mass terms and the long range $\sigma_{n}^{z} \sigma_{m}^{z}$ interactions. The exponentials of $H_{e(o)}$ can be easily written as exact MPO [28]. For $H_{z}$, instead, the exponential can only be approximated. Adopting a second-order Trotter expansion and, for $H_{z}$, a first-order Taylor expansion, we can write

$$
\rho(\beta) \approx\left[e^{-\frac{\delta}{2} H_{e}}\left(1-\frac{\delta}{2} H_{z}\right) e^{-\delta H_{o}}\left(1-\frac{\delta}{2} H_{z}\right) e^{-\frac{\delta}{2} H_{e}}\right]^{M},
$$

where $\delta=\beta / M$ is the step width, and the final error for fixed $\beta$ will be $\mathcal{O}(\delta)$, dominated by the Taylor expansion.
Starting from the identity operator, which corresponds to infinite temperature, $\rho(\beta=0)$, we apply successive Euclidean evolution steps. After each of them a truncation is carried out to find an MPO approximation to the result. To this end, using a Choi isomorphism [31], the MPO is mapped to an MPS with local physical dimension $d^{2}$, and an alternating least squares procedure is applied to minimize the Euclidean distance between the vectorized MPO for the new and evolved states. Since the truncation does not preserve the positivity of the whole state, it is more convenient to compute $\rho(\beta / 2)^{\dagger} \rho(\beta / 2)$, where the Trotter expansion explained above is used for each factor [32]. More details about the method can be found in the Appendix.

\section{RESULTS}

To compute the chiral condensate in the infinite volume and continuum limits, we approximate the thermal state at each $g \beta \in[0,6]$ over a range of values of $x \in[4,65]$. For each value, we consider various system sizes, with $N / \sqrt{x} \in[16,24]$ to ensure consistent physical volumes, and for each of them, different step widths, $\delta$, and bond dimensions. We thus need to control effects of successively extrapolating in $D, \delta, N$, and $x$.

The limited bond dimension, $D$, used for each fixed set of values $(x, N, \delta)$ induces a systematic truncation error. The MPS family being complete, the results converge to the exact value for the given problem in the limit of very large $D$ (of the order of the dimension of the operator space) [23]. From our finite $D$ results, we estimate the final value of $\Sigma$ as the one obtained for the largest achieved bond dimension, $D_{\max }$, and the error as the difference between this value and the one obtained from $D_{\max }-20$, as illustrated in the left panel of Fig. 1.

A second source of systematic error is the finite step width, $\delta$. Although we use a second-order Suzuki-Trotter expansion, the Taylor approximation in (2) induces linear
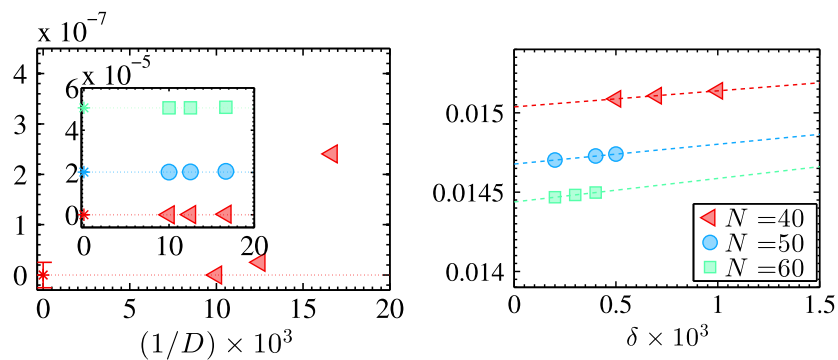

FIG. 1 (color online). Left: convergence of condensate value with bond dimension, $D$, for $g \beta=0.4, x=6.25, N=40$ and $\delta=5 \times 10^{-4}$. Shown is the deviation with respect to the final value, $\Sigma=0.01508769$. The inset shows additionally the results for $\delta=7 \times 10^{-4}$ (circles) and $10^{-3}$ (squares) on a common scale, to compare the magnitude of $D$ and $\delta$ errors. Right: linear $\delta$ extrapolation for the same $x$ and $g \beta$ and several system sizes. 
corrections, $\mathcal{O}(\delta)$. We can thus extrapolate linearly to obtain the value as $\delta \rightarrow 0$, as illustrated by the right panel in Fig. 1 for selected examples. Furthermore, the Taylor approximation requires that the value of $\delta$ employed in the calculation is below a certain threshold, to ensure convergence of the expansion. We find that values $\delta=5 \times 10^{-6}-10^{-3}$ are sufficiently small for the considered values of $x$ and $N$.

The previous steps yield a result for each pair $(x, N)$. As in $[3,4]$, we then find the thermodynamic limit by fitting the results to a linear function in $1 / N$. The left panel of Fig. 2 shows how accurately this extrapolation fits our results for the considered values of $x$.

From the infinite volume results for each lattice spacing at fixed $g \beta$, we can perform the continuum extrapolation. As we showed in [4], the condensate exhibits logarithmic corrections $\mathcal{O}(a \log (a))$. Hence we try two fitting functions, which additionally include linear or linear and quadratic corrections in $a$,

$$
\begin{aligned}
& f_{1}(x)=\Sigma_{\text {cont }}+\frac{a_{1}}{\sqrt{x}} \log (x)+\frac{b_{1}}{\sqrt{x}}, \\
& f_{2}(x)=\Sigma_{\text {cont }}+\frac{a_{2}}{\sqrt{x}} \log (x)+\frac{b_{2}}{\sqrt{x}}+\frac{c_{2}}{x} .
\end{aligned}
$$

To include the uncertainty from the choice of the functional form of the fitting Ansatz, we finally take as central value the result from the fit to $f_{1}$, and as systematic error the difference between both. The right panel of Fig. 2 demonstrates these fits for temperature $g \beta=4.0$. We found that quadratic corrections will only be significant at lower temperatures. Higher order corrections do not provide any significant improvement for the fitting range $x \in[4,65]$.

After performing for each value of $g \beta$ all the steps described above, we obtain for the chiral condensate the temperature dependence shown in Fig. 4. Comparing to the analytical result in [15], we find excellent agreement for all $g \beta \geq 0.5$. Although the central values lie very close to the exact results, the errors shown in Fig. 4 seem relatively large because they include the propagated errors from the
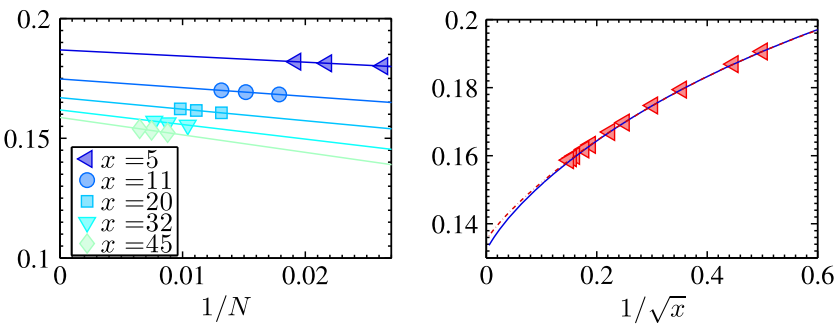

FIG. 2 (color online). Infinite volume (left) and continuum (right) extrapolations of the condensate, $\Sigma$, at $g \beta=4.0$. In the right panel, extrapolations using $f_{1}$ (solid blue) and $f_{2}$ (dashdotted red curve) are shown. extrapolations in $D, \delta, N$, and $x$, as well as the systematic error from the form of the fitting Ansatz for the continuum extrapolation. Our approach makes it possible to fully control all sources of uncertainties. This rigorous account of errors is crucial to ensure that the technique can be used in a general situation, for which no analytical results are available.

Different kinds of errors contribute distinctly at different temperatures. For small $g \beta$, cutoff effects are enhanced, and systematic errors from the choice of the fitting Ansatz can be an order of magnitude larger than other errors. Lowering the temperature, the effect becomes smaller, while other errors grow, in particular the propagated error from the $D \rightarrow \infty$ extrapolation. Interestingly, at intermediate values, $g \beta \approx 2$, the slope of the $N \rightarrow \infty$ extrapolations changes sign. Moreover, for this region of temperatures, the continuum limit extrapolations from $f_{1}$ and $f_{2}$ are very close. For larger $g \beta$, all errors grow, but the increase is faster for $D$ and $\delta$ extrapolations so that at the end of our $g \beta$ range they are an order of magnitude larger than the systematic error from the choice of the fitting Ansatz.

The approach is systematically improvable, because it is clear how to reduce each uncertainty. For the present analysis, the relatively most important error comes from the extrapolation in $D$. We chose a rather conservative criterion to estimate this error and it is natural to expect that much more accurate results can be obtained by checking the convergence in bond dimension with larger values of $D$. The cost of the computation scales with $D^{3}$, which makes the scan over a rather broad range of $D$ feasible. It is nevertheless remarkable that the very accurate results presented here were obtained with a relatively small $D \leq 100$. This shows how adequate the MPO Ansatz is for thermal states in this gauge theory.

For the region of very small $g \beta$, we find a significant deviation from the analytical results (see Fig. 4), although the individual points at finite $x$ are accurate enough, because much smaller lattice spacings are required in order to correctly capture the asymptotic behavior. Using the procedure described above, it is possible to reach larger values of $x$ by incurring a higher computational cost, since the required system size grows as $\sqrt{x}$ to maintain a consistent physical volume, and correspondingly the threshold $\delta$ that ensures convergence decreases.

Using an alternative approximation for the exponential of $H_{z}$ that avoids the Taylor expansion, it is however possible to explore the region of larger $x$ at a lower computational cost. Indeed, $e^{-\delta H_{z}}$ can be written exactly as an MPO of bond dimension $\mathcal{O}(N)$. For systems of several hundreds of sites this is unpractical, but this exponential can be approximated by projecting out those spin configurations that correspond to an electric flux larger than a certain cutoff, $L_{\text {cut }}$, on any of the links. This results in an MPO with bond dimension $2 L_{\text {cut }}+1$. Notice that this truncation is equivalent to limiting the maximum 

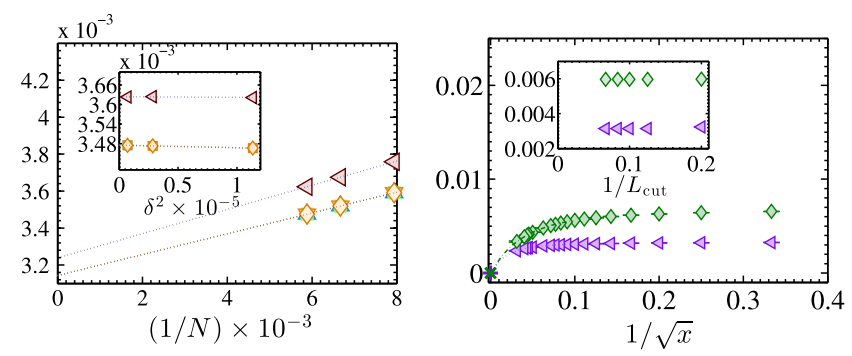

FIG. 3 (color online). Condensate values with a truncation $L_{\text {cut }}$ of the maximum electric flux per link. Left: finite volume extrapolation for $x=55, g \beta=0.1$ from $L_{\text {cut }}=5$ (left-pointing red triangles), 8, 10, 12 and 15 (different shapes and colors, indistinguishable in the plot). The inset shows the extrapolation in Trotter parameter, $\delta$, for $N=170$ and $L_{\text {cut }}=8-15$. Right: continuum limit for $g \beta=0.1$ (left-pointing purple triangles) and 0.2 (green diamonds). The corresponding exact results from [15] are indicated on the vertical axis. The inset shows explicitly the dependence on the cutoff for these $g \beta$ values at $x=55$.

occupation number for the bosonic degrees of freedom, as done in $[1,5,6]$ for pure states. However, the latter results in twice as large system sizes, since bosonic degrees of freedom are kept explicitly, and, in principle, in local dimensions that scale as $\left(2 L_{\text {cut }}+1\right)^{2}$ for the additional sites in the MPO [33].

Although the cost of applying the MPO for the projected exponential is higher than that of the Taylor approximation, the step width error is now $\mathcal{O}\left(\delta^{2}\right)$, determined by the second-order Trotter expansion, and there is no threshold value for $\delta$, which allows us to reach the same $g \beta$ with fewer steps. To explore the small $g \beta$ region, we study the range $x \in[4,1024]$. For each value of $x$, we compute different system sizes, step widths and $L_{\text {cut }}$ values, and for each of them bond dimensions up to $D=160$. As described above, we successively extrapolate $1 / D \rightarrow 0$ (as before), $\delta \rightarrow 0$ (linear in $\delta^{2}$ ) and $1 / N \rightarrow 0$, as illustrated by Fig. 3 (left). To account for the additional systematic error due to the cutoff parameter, we can also extrapolate in $L_{\text {cut }}$. However, comparing the results for $L_{\text {cut }} \in[5,15]$, we observe (see Fig. 3) that the effect is very small, and results for $L_{\text {cut }} \geq 8$ are compatible within our numerical precision (inset of Fig. 3, right), so here we present the results for $L_{\text {cut }}=10$ and leave the detailed analysis of the cutoff effects to a more technical work [35].

This relatively small $L_{\text {cut }}$ allows us to study the lattice effects for the smallest $g \beta$. As shown in Fig. 3, $g \beta=0.1$ requires $x \approx 300$ or larger to obtain an accurate continuum extrapolation. We observe that higher order corrections are present and adopt as central value the result of the fit $f_{3}(x)=\Sigma_{\text {cont }}+\frac{a_{3}}{\sqrt{x}} \log (x)+\frac{b_{3}}{\sqrt{x}}+\frac{c_{3}}{x}+\frac{d_{3}}{x \sqrt{x}} \quad$ [36] and we estimate the error from using different fitting ranges. Notice that to properly deal with the uncertainty due to the fit, we could run a statistical analysis as was done in [3], but the simple estimate used here allows us to appreciate the relevance of reaching large $x$ values.
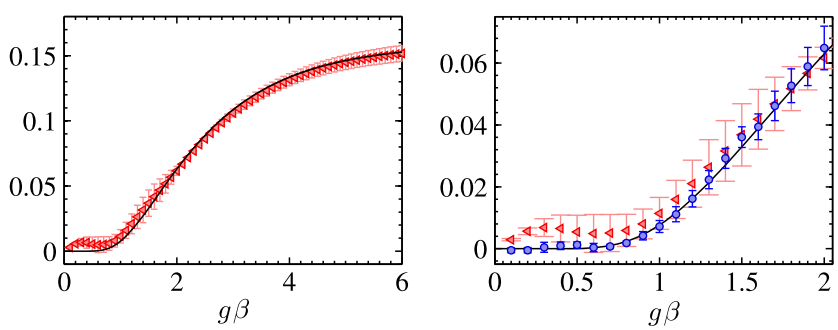

FIG. 4 (color online). Temperature dependence of the chiral condensate from data with $x \leq 65$ and exact gauge sector (red triangles), compared to [15] (solid line). For the lowest $g \beta$ (right), the results deviate from the exact ones. Using the $L_{\text {cut }}=10$ truncation, we reach smaller lattice spacings and recover the consistency with the analytical results (blue circles).

Finally, we obtain for the temperature dependence of the chiral condensate the improved results shown in Fig. 4 (right), consistent with the analytical result at high temperatures.

\section{CONCLUSION}

Using the massless Schwinger model as a benchmark, we have demonstrated for the first time that finite matrix product operators can successfully describe thermal equilibrium states of lattice gauge theories. This complements earlier works that proved the suitability of MPS for describing the low energy states of the spectrum. We have evaluated the thermal evolution of the chiral condensate in this model and found good agreement with the analytical result [15] from infinite to almost zero temperature. The high temperature region is the hardest one to capture in the continuum limit, maybe counterintuitively, since for typical condensed matter models it is easier to describe. On the other hand, it is known that lattice spacing effects in the high temperature region can be non-negligible in conventional lattice simulations. We have nevertheless shown that, using the MPO Ansatz, it is possible to obtain precise results even at very small lattice spacings.

Our approach offers a systematic procedure to evaluate and control all systematic errors in the calculation, namely the bond dimension of the Ansatz, step width of the Trotter expansion for the exponential operators, finite volume and continuum limit. Although not strictly necessary, a truncation of the maximum electric flux per link can be introduced to enhance the numerical performance. The effect of this additional cutoff parameter is very small, but can equally be taken into account in the systematic error analysis.

All this makes the MPS and MPO Ansätze very valuable and promising tools to evaluate also other one-dimensional Hamiltonian systems relevant for gauge field theories. The most interesting open question is the extension of these techniques to higher dimensions. 


\section{ACKNOWLEDGMENTS}

We are grateful to B. Buyens, S. Kühn, M. Lubasch and K. Van Acoleyen for discussions. This work was partially funded by the EU through SIQS Grant No. FP7 600645, and by the DFG Sonderforschungsbereich/Transregio Grant No. SFB/TR9. K. C. has been supported in part by the Helmholtz International Center for FAIR within the framework of the LOEWE program launched by the State of Hessen. H. S. was supported by the Japan Society for the Promotion of Science for Young Scientists. We are grateful to the computer centers of DESY Zeuthen, RZG Garching and the Center for Scientific Computing of the GoetheUniversity in Frankfurt (the LOEWE system) for their computing resources and support.

\section{APPENDIX: NUMERICAL METHOD}

For completeness, we describe here the details of the numerical method used in this paper.

To describe the thermal equilibrium state we use the MPO Ansatz [26-28],

$$
\hat{O}=\sum_{\left\{i_{k}, j_{k}\right\}} \operatorname{tr}\left(M_{0}^{i_{0} J_{0}} \cdots M_{N-1}^{i_{N-1} j_{N-1}}\right)\left|i_{0} \cdots i_{N-1}\right\rangle\left\langle j_{0} \cdots j_{N-1}\right| .
$$

The thermal density operator can be written as imaginary evolution of the identity operator [26], $\rho(\beta) \propto e^{-\beta H}=$ $e^{-\frac{\beta}{2} H} \mathbb{1} e^{-\frac{\beta}{2} H}$. By applying a Choi isomorphism [31], $|i\rangle\langle j|\rightarrow| i\rangle \otimes|j\rangle$, the operators can be vectorized. The thermal state can thus be approximated as an MPS, by applying the imaginary time evolution operator corresponding to $\tilde{H}=H \otimes \mathbb{1}+\mathbb{1} \otimes H^{T}$ on the initial vectorized identity (see Fig. 5). As shown in the main text, the Hamiltonian contains noncommutative terms, so we approximate the exponential operator as a sequence of MPOs, to be applied on the MPS. To this end, we use a second-order Suzuki-Trotter expansion,

$$
e^{-\beta \tilde{H}} \approx\left[e^{-\frac{\delta}{2} \tilde{H}_{e}} e^{-\frac{\delta}{2} \tilde{H}_{z}} e^{-\delta \tilde{H}_{o}} e^{-\frac{\delta}{2} \tilde{H}_{z}} e^{-\frac{\delta}{2} \tilde{H}_{e}}\right]^{M} .
$$

Each evolution step is thus approximated as the successive action of five terms. The exponentials of the hopping terms, $H_{e(o)}=\sum_{n \text { even(odd) }}\left(\sigma_{n}^{+} \sigma_{n+1}^{-}+\right.$H.c. $)$, have a simple exact

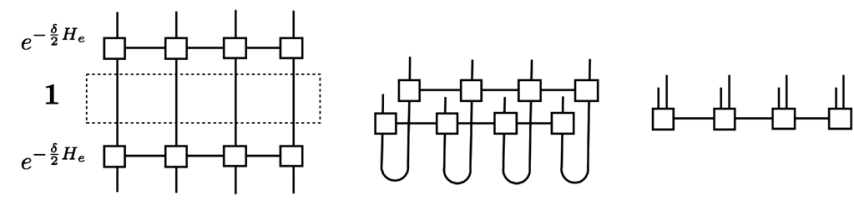

FIG. 5. Schematic representation of the TN operations. Left: applying the first exponential for the first step of imaginary time evolution on the identity operator (which is an MPO with $D=1$ ). Center: vectorizing this first step allows us to work with the MPS formalism. Right: after each application of the exponential, the result is approximated by an MPS with bounded bond dimension.
MPO expression with maximal bond dimension 4 [28], constructed as simply the product of the individual exponentials of the mutually commuting two-body terms. The remaining term,

$$
\begin{aligned}
H_{z}= & \frac{\mu}{2} \sum_{n=0}^{N-1}\left[1+(-)^{n} \sigma_{n}^{z}\right] \\
& +\frac{1}{4} \sum_{n=0}^{N-2}\left[(n+1)+2 \sum_{k=0}^{n} \sum_{l<k} \sigma_{k}^{z} \sigma_{l}^{z}\right] \\
& +\sum_{n=0}^{N-2}\left(1+2 \sum_{k=0}^{n} \sigma_{k}^{z}\right),
\end{aligned}
$$

(even)

contains long-range terms $\sigma_{n}^{z} \sigma_{m}^{z}$, and, although all terms commute with each other, the product of individual exponentials would yield a bond dimension exponentially large with the system size, $N$.

A more efficient expression for the exponential exists with bond dimension that only scales linearly in $N$. We can indeed write $H_{z}$ as a sum of mutually commuting local terms, $H_{z}=\sum_{n} h_{n}$, where, for $n<N-1$,

$$
h_{n}=\frac{\mu}{2}\left[1+(-1)^{n} \sigma_{n}^{z}\right]+L_{n}^{2},
$$

and $h_{N-1}=\frac{\mu}{2}\left[1+(-1)^{N-1} \sigma_{N-1}^{z}\right], L_{n}$ being the electric flux on each link. The exponential of $H_{z}$ is diagonal in the $z$ spin basis, and its value on a basis vector can be written as a product of the exponentials of each of these terms for the corresponding state. Since the value of $L_{n}$, by virtue of Gauss' law, is completely determined by the spin content on sites $k \leq n$, the factor corresponding to a given link can be determined from the total magnetization, $\sum_{k \leq n} \sigma_{k}^{z}$, to the left of the corresponding site. Such information can be encoded in the virtual index of an MPO, which, in a chain of length $N$, could in principle assume values $L_{n} \in[-N / 2, N / 2]$. The exponential can thus be written exactly as an MPO determined by tensors $M_{n}$ whose only nonvanishing elements are $\left(M_{n}^{i i}\right)_{L_{n-1} L_{n}}=e^{-\delta h_{n}}$ for $L_{n}=$ $L_{n-1}+\frac{1}{2}\left[(-1)^{n}+\left(\sigma_{n}^{z}\right)_{i i}\right]$.

Such exact expression produces an MPO with bond dimension $\mathcal{O}(N)$, which is not practical for the long chains involved in our study. Thus, it is convenient to truncate the MPO by allowing $L_{n}$, i.e. the virtual index, to assume only bounded values $\left|L_{n}\right| \leq L_{\text {cut }}$, so that the maximum bond dimension is $2 L_{\text {cut }}+1$. This corresponds to a truncation of the physical space to only those spin configurations for which all links have small enough electric flux, since the rest will be projected out when multiplying by the truncated exponential.

Another, more economical approximation to the exponential of $H_{z}$ can be achieved by a first-order Taylor expansion, which can be written as an MPO with bond dimension 3. In this approach, the whole physical space is 
kept, so that no extrapolation in the $L_{\text {cut }}$ parameter is required. In our calculation, we use both approaches.

The exponentials in (A2) involve $\tilde{H}_{\alpha}=H_{\alpha} \otimes \mathbb{1}+\mathbb{1} \otimes$ $H_{\alpha}^{T}$ for each $\alpha=e, o, z$. But since both terms in each $\tilde{H}_{\alpha}$ commute, the corresponding exponential is just the tensor product of two exponentials, which can then be applied sequentially or simultaneously. After every factor in (A2) is written or approximated as an MPO, the effect of one evolution step on a certain intermediate state, vectorized as an MPS, can be approximated as a new MPS with the desired bond dimension. This is achieved by a global optimization (see, e.g., [37] for algorithmic details), in which the Euclidean distance $\epsilon=\|\left|\rho^{\prime}\right\rangle-O|\rho\rangle \|^{2}$ between the new MPS, $\left|\rho^{\prime}\right\rangle$, and the result of the operator $O$ on the original one, $|\rho\rangle$, is minimized by successively varying each one of the tensors, and sweeping over the chain until convergence (Fig. 5).
[1] T. Byrnes, P. Sriganesh, R. Bursill, and C. Hamer, Phys. Rev. D 66, 013002 (2002).

[2] K. Cichy, A. Kujawa-Cichy, and M. Szyniszewski, Comput. Phys. Commun. 184, 1666 (2013).

[3] M. Bañuls, K. Cichy, K. Jansen, and J. Cirac, J. High Energy Phys. 11 (2013) 158.

[4] M. C. Bañuls, K. Cichy, J. I. Cirac, K. Jansen, and H. Saito, Proc. Sci., LATTICE2013 (2014) 332 [arXiv:1310.4118].

[5] B. Buyens, K. Van Acoleyen, J. Haegeman, and F. Verstraete, Proc. Sci., LATTICE2014 (2014) 308 [arXiv: 1411.0020].

[6] B. Buyens, J. Haegeman, K. Van Acoleyen, H. Verschelde, and F. Verstraete, Phys. Rev. Lett. 113, 091601 (2014).

[7] E. Rico, T. Pichler, M. Dalmonte, P. Zoller, and S. Montangero, Phys. Rev. Lett. 112, 201601 (2014).

[8] P. Silvi, E. Rico, T. Calarco, and S. Montangero, New J. Phys. 16, 103015 (2014).

[9] Y. Shimizu and Y. Kuramashi, Phys. Rev. D 90, 014508 (2014).

[10] Y. Shimizu and Y. Kuramashi, Phys. Rev. D 90, 074503 (2014).

[11] L. Tagliacozzo, A. Celi, and M. Lewenstein, Phys. Rev. X 4, 041024 (2014).

[12] J. Haegeman, K. Van Acoleyen, N. Schuch, J. I. Cirac, and F. Verstraete, Phys. Rev. X 5, 011024 (2015).

[13] S. Borsanyi, G. Endrodi, Z. Fodor, S. Katz, and K. Szabo, J. High Energy Phys. 07 (2012) 056.

[14] U. Heinz, P. Sorensen, A. Deshpande, C. Gagliardi, F. Karsch et al., arXiv:1501.06477.

[15] I. Sachs and A. Wipf, Helv. Phys. Acta 65, 652 (1992).

[16] H. Saito, M. C. Bañuls, K. Cichy, J. I. Cirac, and K. Jansen, Proc. Sci., LATTICE2014 (2014) 302 [arXiv:1412.0596].

[17] J. Schwinger, Phys. Rev. 128, 2425 (1962).

[18] J. Kogut and L. Susskind, Phys. Rev. D 11, 395 (1975).
[19] T. Banks, L. Susskind, and J. B. Kogut, Phys. Rev. D 13, 1043 (1976).

[20] C. J. Hamer, Z. Weihong, and J. Oitmaa, Phys. Rev. D 56, 55 (1997).

[21] F. Verstraete, D. Porras, and J. I. Cirac, Phys. Rev. Lett. 93, 227205 (2004).

[22] G. Vidal, Phys. Rev. Lett. 93, 040502 (2004).

[23] D. Perez-García, F. Verstraete, M. M. Wolf, and J. I. Cirac, Quantum Inf. Comput. 7, 401 (2007).

[24] M. B. Hastings, J. Stat. Mech. (2007) P08024.

[25] U. Schollwöck, Ann. Phys. (Amsterdam) 326, 96 (2011).

[26] F. Verstraete, J. J. García-Ripoll, and J. I. Cirac, Phys. Rev. Lett. 93, 207204 (2004).

[27] M. Zwolak and G. Vidal, Phys. Rev. Lett. 93, 207205 (2004).

[28] B. Pirvu, V. Murg, J. I. Cirac, and F. Verstraete, New J. Phys. 12, 025012 (2010).

[29] M. Hastings, Phys. Rev. B 73, 085115 (2006).

[30] A. Molnar, N. Schuch, F. Verstraete, and J. I. Cirac, Phys. Rev. B 91, 045138 (2015).

[31] M.-D. Choi, Linear Algebra Appl. 10, 285 (1975).

[32] Notice that this is equivalent to the purification Ansatz.

[33] By using symmetric tensors that enforce Gauss' law, the squaring of local bosonic dimensions can nevertheless be avoided [34].

[34] B. Buyens (private communication).

[35] M. C. Bañuls, K. Cichy, J. I. Cirac, K. Jansen, and H. Saito (to be published).

[36] For the smallest $g \beta \leq 0.5$, we use $x \geq 100$, for $0.5<g \beta \leq 1.5, x \geq 100$, and for larger $g \beta, x \geq 9$.

[37] F. Verstraete, V. Murg, and J. Cirac, Adv. Phys. 57, 143 (2008). 